\title{
Science avec patience
}

\section{Georges Didi-Huberman}

\section{OpenEdition}

\section{Journals}

Édition électronique

URL : http://journals.openedition.org/imagesrevues/3024

DOI : 10.4000/imagesrevues.3024

ISSN : 1778-3801

\section{Éditeur :}

Centre d'Histoire et Théorie des Arts, Groupe d'Anthropologie Historique de l'Occident Médiéval, Laboratoire d'Anthropologie Sociale, UMR 8210 Anthropologie et Histoire des Mondes Antiques

\section{Référence électronique}

Georges Didi-Huberman, « Science avec patience », Images Re-vues [En ligne], Hors-série 4 | 2013, mis en ligne le 30 janvier 2013, consulté le 31 janvier 2021. URL : http://journals.openedition.org/ imagesrevues/3024; DOI : https://doi.org/10.4000/imagesrevues.3024

Ce document a été généré automatiquement le 31 janvier 2021.

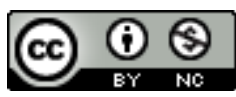

Images Re-vues est mise à disposition selon les termes de la Licence Creative Commons Attribution Pas d'Utilisation Commerciale 4.0 International. 


\title{
Science avec patience
}

\author{
Georges Didi-Huberman
}

1 Le 2 novembre 1918 - soit à l'approche d'une capitulation allemande qui devait mettre fin au premier conflit mondial, laissant en Europe pas moins de neuf millions de morts et vingt et un millions de blessés, d'estropiés, de traumatisés -, à quatre heures du matin, Aby Warburg fut admis d'urgence dans la clinique du docteur Arnold Lienau, à Hambourg, après avoir - revolver en main, hurlant, hors de

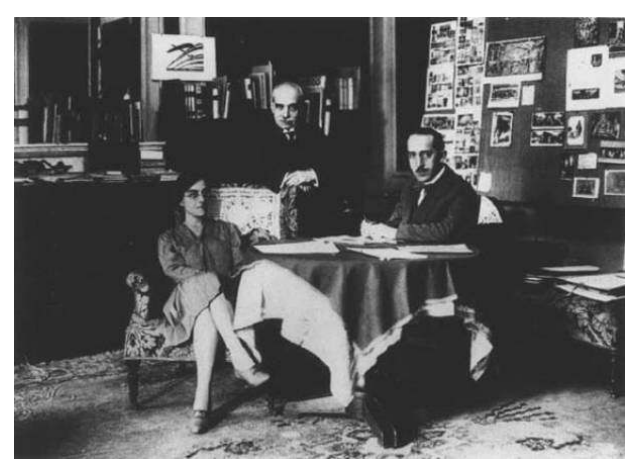
lui - menacé la vie de ses proches et la sienne propre; on lui administra immédiatement toute une série de substances médicamenteuses telles que le Pantopon, le Tropfen ou le Veronal ${ }^{1}$. Mais il en fut de cette crise comme de la guerre elle-même: ce n'était pas un épisode mais bien un processus, qui devait tenir l'historien des images entre les murs de différents asiles jusqu'en 1924. La guerre était, certes, terminée en tant qu'épisode historique ; mais la psychomachie mémorielle continuait, avec son poids de douleurs toujours plus lourdes sur les épaules de notre Atlas moderne. Heinrich Embden, le médecin traitant de Warburg, décrivait ainsi la « chute » de 1918 :

«De graves symptômes semblent s'être manifestés d'une manière relativement immédiate à l'automne 1918, sous l'effet d'impressions produites par notre situation désespérée. (J'étais alors au front.) Comme je l'ai déjà rapporté oralement, il croyait qu'une gouvernante anglaise, amie de sa famille, qui était restée à Hambourg durant les premiers mois de la guerre, avait été "l'espionne en chef de Lloyd George" et que lui, Warburg, serait par conséquent rendu responsable de l'issue malheureuse de la guerre et puni pour cela. D'une heure à l'autre, il s'attendait à une catastrophe (emprisonnement, etc.), et l'agitation inhérente à un tel complexe conduisit au premier fait marquant de sa psychose - il menaça sa famille avec un revolver, pour la préserver du pire en la tuant - puis à son transfert à la clinique. Ici, ses hallucinations, très vives, ont eu un caractère presque exclusivement menaçant et angoissant. Les voix se tournaient contre lui et contre sa famille. Il entendait que l'on tirait des coups de feu sur sa femme et répondait 
dans une extrême agitation à ses appels au secours. En outre, délire de préjudice à caractère physico-chimique : peur des métaux et des objets de métal, à cause de l'influence électrique ; peur de l'empoisonnement, parce que l'eau du bain contenait du sublimé ${ }^{2}$ [chlorure de mercure]. »

L'« anamnèse » clinique rédigée le 19 mai 1921 par Heinrich Embden ne laisse aucun doute sur le fait que ce désastre psychique d'Aby Warburg se présentait d'abord comme un désastre de la guerre :

«La guerre plongea W[arburg] dans une agitation démesurée (maßlos), en partie à cause de ses sentiments patriotiques, élevés et purs, en partie à cause des répercussions personnelles qu'elle provoquait en lui. Il eut très tôt une juste intuition des périls, après la bataille de la Marne. Il jouait avec l'idée de s'engager comme interprète, il en parlait beaucoup : "C'est un poste où l'on peut facilement prendre une balle." Prit des cours d'équitation, se munit de bottes de campagne et de guêtres, [...] essaya, grâce à de vieilles relations, de travailler pour la patrie, plus particulièrement à l'Institut allemand d'Histoire de l'Art, à Florence. [...] Pendant les années de guerre, est devenu toujours plus agité. Assembla une énorme collection de journaux, lisant sept quotidiens, soulignant tout ce qui concernait l'actualité ; tout cela fut catalogué, dans une gigantesque cartothèque, par un groupe de collaborateurs. Par ailleurs, menait des recherches toujours plus poussées sur la superstition. Pour son principal projet scientifique, la survivance des modes de pensée antiques au Moyen Âge, il s'était consacré à l'étude de l'astrologie, etc. Alors, il glissa peu à peu du point de vue de l'historien vers une demi-croyance, puis un comportement superstitieux. [...] Il en était venu à se prendre pour un loup-garou. Il croyait ne pouvoir se soustraire à des malheurs imminents qu'en tuant sa famille et en se suicidant; il se saisit d'un revolver, fut facilement désarmé et, dans les premiers jours de novembre 1918, on le conduisit à la clinique du docteur Lienau ${ }^{3}$.»

3 On sait qu'après les services psychiatriques de Hambourg et d'Iéna, c'est à la clinique Bellevue de Kreuzlingen qu'Aby Warburg finit par être admis, le 16 avril 1921, et qu'il y suivit, sous la responsabilité de Ludwig Binswanger, une longue cure ${ }^{4}$ marquée par la célèbre conférence sur Le Rituel du serpent - prononcée devant un public de savants et de fous - à l'issue de laquelle l'interminable psychomachie aura pris le visage d'une interminable guérison de l'âme, cette unendliche Heilung dont Davide Stimilli a voulu intituler sa remarquable édition de l'histoire clinique d'Aby Warburg ${ }^{5}$. On sait aussi les difficultés rencontrées par les psychiatres pour nommer la souffrance dont l'historien était atteint : Binswanger prononça d'abord un diagnostic de schizophrénie qui excluait toute reconstruction intellectuelle $d u$ patient ( je tiens qu'une reprise $d u$ travail scientifique est des plus improbable ", écrivait-il à Embden le 18 août 19216), avant de se ranger à l'opinion émise par Emil Kraepelin, un diagnostic d'« état mixte maniacodépressif » agrémenté d'un « pronostic absolument favorable » pour le retour au travail de la pensée ${ }^{7}$.

Ces débats diagnostiques autour du cas de Warburg nous fournissent l'indication que le problème de sa folie ne saurait être réduit à l'observation d'un "défaut " et à sa conceptualisation "séméiologique ", bref à son enchâssement unilatéral dans le cadre d'un «tableau clinique». Il faut évidemment prendre au sérieux les approches psychopathologiques - subtiles et compréhensives - de Binswanger à l'égard de son patient, mais il faut également rester à l'écoute du patient lui-même en tant qu'être pensant. Si Warburg a tant parlé de "psychomachies» dans ses études d'histoire culturelle, ne faut-il pas aussi le prendre au sérieux de son désastre psychique en tant que symptôme d'une tragédie de la culture qui se jouait au-delà de lui, tout autour de 
lui, depuis le début de la Grande Guerre ? On sait le rôle considérable du concept de Denkraum dans l'œuvre de Warburg: cet « espace de la pensée » tour à tour construit (dans les résultats de sa science historique) et détruit (dans les décombres de la guerre), reconstruit (dans les images et les fiches de sa cartothèque) et redétruit encore (dans l'effondrement de novembre 1918)...

On s'aperçoit ainsi, à lire l'histoire clinique de Warburg, que pas un seul de ses motifs délirants n'est, en fait, séparable des grands paradigmes où s'organisait depuis longtemps sa pensée historique et philosophique. La folie de Warburg fut donc, d'abord, un destin de son Denkraum. Sa "psychomachie », une lutte menée dans l'espace de la pensée entre les astra et les monstra, les constructions pour recueillir la multiplicité du monde et les explosions de ce même monde en millions de cadavres (la guerre réelle) et en fantômes efficaces (la guerre dans l'âme). Dès le début de son internement psychiatrique, par exemple, Aby Warburg aura éprouvé une parenté directe - et légitime - avec le cas de Friedrich Nietzsche soigné quelques années plus tôt par un certain Ludwig Otto Binswanger, l'oncle de son propre médecin à Kreuzlingen ${ }^{8}$. Nous ne devrons donc pas craindre de reconnaître, à l'autre bout de ce processus, l'atlas Mnémosyne lui-même comme un moment décisif de cette grande " psychomachie » tout à la fois singulière et impersonnelle, ce remontage final d'un Denkraum déséquilibré par les désastres de la Grande Guerre.

6 Ainsi, dans sa longue lettre de 1921 au personnel médical de la clinique Bellevue - un nom prédestiné, semble-t-il -, Aby Warburg écrivait de lui-même: «Ma maladie consiste en ce que je perds la capacité de relier les choses d'après leurs simples rapports de causalité (daß ich die Fähigkeit, die Dinge in ihren einfachen Kausalitätsverhältnissen zu verknüpfen, verlierte), ce qui se reflète dans le domaine spirituel aussi bien que réel ${ }^{9} . .$. » Bien qu'il soit, dans la suite de cette phrase, question d'« aubergines farcies d'une manière indéfinissable» - et de la surinterprétation délirante qui en pourra résulter -, c'est bien le logos même et l'épistémè du grand historien qui s'énoncent ici en toute lucidité. Mnémosyne nous montre bien, en effet, que le génie de Warburg consistait justement en ceci qu'il était capable de relier les images par-delà leurs « simples rapports de causalité ».

7 Il n'est pas fortuit qu'en 1927, soit à une époque d'intense travail sur l'atlas Mnémosyne, Aby Warburg ait consacré un séminaire particulier au "gai savoir inquiet» de l'historien. Or, il voulut l'incarner dans le binôme constitué par Jacob Burckhardt et Friedrich Nietzsche, pour le focaliser aussitôt sur le point même où l'inquiétude se fait déséquilibre, à savoir l'effondrement psychique de Nietzsche en 1889. C'est que, selon Warburg, les historiens ne sauraient être réduits au simple statut de chroniqueurs $\mathrm{du}$ temps qui passe : ils sont d'abord des «récepteurs d'ondes mnémiques (Auffänger der mnemischen Wellen), [...] des sismographes très sensibles (sehr empfindliche Seismographen) dont tremblent les fondations lorsqu'ils doivent capter l'onde et la transmettre »; d'où « le risque (Gefährlichkeit) que comporte [cette] profession, celui d'un effondrement pur et simple ${ }^{10}$. " Devant ce danger ou cette inquiétude fondamentale, Burckhardt se sera remparé dans une "tour d'ivoire» faite de livres, d'images et de fiches (comme Warburg dans sa bibliothèque); mais Nietzsche, dans la lumière de Turin, aura fait de cette inquiétude un déséquilibre fatal, une chute dans la folie (comme Warburg dans ses crises).

8 L'auteur de Mnémosyne concluait que Burckhardt est un voyant qui parvient à demeurer fidèle aux grandes lucidités des Lumières, tandis que Nietzsche est un visionnaire du 
type nabi, "l'ancien prophète qui court à travers rues, déchire ses vêtements, se lamente et, parfois, entraîne le peuple à sa suite ${ }^{11}$. » Il est aisé de comprendre, en lisant ce séminaire subtilement autobiographique, que Warburg fut tout cela à la fois : un voyant du temps animé par une psychomachie constante des astra (en tant qu'homme des Lumières, philologue précis, collectionneur de livres, de fiches et d'images) avec les monstra (en tant qu'homme tragique, philosophe inspiré, visionnaire halluciné des «ondes mnémiques " produites par les séismes de l'histoire). Voilà pourquoi le récit clinique d'Aby Warburg doit lui-même se lire selon la double optique des astra et des monstra, comme si l'espace hallucinatoire de ses visions délirantes n'était que l'explosion - éclat devenu éclatement - d'un espace de pensée malgré tout, sa propre vision de l'histoire. C'est-à-dire une version, mais "démontée ", de sa connaissance la plus authentique. Nietzsche n'avait-il pas, dès Aurore, théorisé les vertus d'une telle connaissance par la souffrance?

"L'être profondément souffrant jette sur les choses, $d u$ fond de son mal, un regard d'une épouvantable froideur : tous ces petits enchantements trompeurs au milieu desquels les choses baignent habituellement lorsqu'elles sont contemplées par l'œil d'un bien-portant ont disparu pour lui : il gît lui-même sous son propre regard, sans charme et sans couleur. À supposer qu'il ait vécu jusque-là dans quelque dangereuse rêverie, le suprême rappel à la réalité de la douleur constitue le moyen de l'arracher à cette rêverie: et peut-être le seul moyen. [...] La monstrueuse tension de l'intellect qui veut tenir la douleur en respect fait que tout ce qu'il regarde désormais s'éclaire d'une nouvelle lumière; et l'indicible attrait qu'exercent toujours les nouveaux éclairages est souvent assez puissant pour mettre en échec toutes les tentations du suicide et pour faire paraître hautement désirable à l'être souffrant de continuer à vivre ${ }^{12}$. "

Cette façon si «vitale» de comprendre le savoir du souffrant pourrait, sans peine, s'appliquer au cas d'Aby Warburg. Lorsque en lui le sismographe eut explosé, les " ondes mnémiques » n'avaient plus à transiter par livres, images et fiches interposées : elles venaient directement bouleverser son âme, sa vision et tous les membres de son corps. Elles le défiguraient pour cela, sans doute. Mais les traces monstrueuses qu'elles laissaient sur sa vie consciente n'en étaient pas moins les traces d'une guerre réelle et impersonnelle qu'il ne faisait, après tout, que subir et convertir en monstra. Warburg à Kreuzlingen fut en ce sens un être du duende, dans l'acception précise, dionysiaque et spectrale, que devait lui donner Federico García Lorca en le dépouillant de toute protection des Muses ${ }^{13}$. Clio n'était plus là, en effet, pour assurer Warburg d'une clarté du récit. Dans le désordre temporel - disparates, caprices ou désastres - qui l'agitait alors, il était le jouet des Érinyes plutôt que des Muses, de Dionysos plutôt que d'Apollon, du pathos plutôt que du logos. Chacun de ses astra, ses constellations de pensée, se décomposait - mis en pièces et révélé en même temps - sous ses figures grouillantes de monstra.

Par exemple, ce qui avait justifié l'exceptionnelle et célèbre précision philologique de Warburg - «le bon Dieu gît dans le détail» - se trouva, après 1918, livré à l'exagération paranoïaque la plus incontrôlable, ce qu'Heinrich Embden nomma une « susceptibilité excessive » (übermäßige Empfindlichkeit) pour les détails : « Il revêtait des choses bénignes d'une signification aiguë, gigantesque, en en faisant une question de principe $^{14}$ » (eine scharfe und großartige prinzipielle Einkleidung). Dans le même temps, son profond respect des singularités - ce principe épistémologique si fécond dans son œuvre -, allié au leitmotiv de la survivance, lui faisait voir une âme dans chaque chose, si modeste fût-elle: "Chaque petit pois, chaque pomme de terre, chaque haricot est 
l'âme d'un homme ${ }^{15}$.» Façon d'être rattrapé par cet «animisme » qu'il avait si souvent étudié d'un point de vue anthropologique, des Grecs anciens jusqu'à la Renaissance en passant par les Indiens Hopi. Plus de Muses, donc, mais des Psychés partout : c'est ainsi que Binswanger, le 2 juillet 1921, consigne dans ses notes que Warburg « s'agite, le soir, lorsque des papillons de nuit, attirés par la lumière, volettent dans sa chambre. A peur qu'ils ne soient tués par le gardien et n'en dort pas pendant des heures; fait part de sa douleur aux papillons ${ }^{16} . »$ Et, en date du 10 août :

«[Warburg] s'est inventé un culte avec les petits papillons de nuit qui volettent dans sa chambre la nuit. Il les appelle "petites bêtes qui ont une âme" (Seelentierchen), il peut s'entretenir avec elles pendant des heures. Est très préoccupé parce que son "petit papillon" n'a rien à manger ; veut lui donner du lait, lui rapporte de sa promenade une feuille de tilleul. Est malheureux quand le petit papillon s'en va. Le cherche alors partout. Est heureux de retrouver un autre petit animal. Il leur parle de la façon suivante : "Petit papillon, le professeur te remercie de pouvoir bavarder avec toi, puis-je te dire toute ma douleur (darf ich dir all mein Leid klagen), pense un peu, petit papillon, le 18 novembre 1918, j'ai eu si peur pour ma famille que j'ai pris mon revolver et que j'ai voulu la tuer, et moi avec. Tu sais, parce que les bolcheviques arrivaient ${ }^{17}$. »

11 Mais, au grand théoricien des polarités, on ne pouvait manquer d'attendre que toute chose versât dans son contraire. Binswanger se dit lui-même impressionné, chez Warburg, par le « contraste frappant entre, d'un côté, son tendre respect des plantes, des animaux et des objets inanimés (particulièrement des emballages, comme ceux des chocolats, qu'il ne fallait pas jeter) et, d'un autre côté, son agressivité intellectuelle, sa

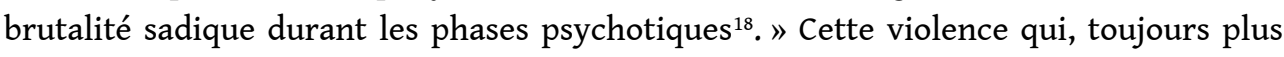
loin des Muses, le faisait ressembler à Saturne - c'est-à-dire à Chronos, le Temps dévorant ses enfants. Même Fritz Saxl, dans ses notes sur Kreuzlingen, se laissera aller à la comparaison: "C'est un rude père saturnien ${ }^{19}$ " (ein harter Saturn-Vater). Cette immense force négative fit hurler Warburg des heures durant - il y perdit définitivement son timbre de voix - et frapper autrui avec, précise Binswanger, une «force colossale ${ }^{20}$ " (kolossale Kräfte), tel Atlas menant seul sa guerre contre tous les dieux de l'olympe.

Cette violence saturnienne ou titanesque n'était, évidemment, que l'autre face d'une terreur de tous les instants. Warburg ne voyait une âme dans chaque chose que parce qu'il voyait une mort en chaque chose ou en chaque image : effet d'une guerre ou d'un meurtre obsidional disposant ses poisons, ses complots, ses armes fatales et ses cadavres tout autour de lui. Warburg, à Kreuzlingen, ce fut d'abord Saturne hanté par l'angoisse d'avoir dévoré - ou de devoir dévorer - sa propre famille, avant d'être mis à mort à son tour : dans les pralines il suspectait la chair de son frère dont il éprouvait l'horreur qu'elle passe dans son ventre puis finisse dans les toilettes; c'est pourquoi, écrit Binswanger, "il laisse nécessairement un reste chaque fois qu'il mange quelque chose. Si par mégarde il mange un de ces restes, il est extrêmement malheureux et se lamente d'avoir dévoré l'un de ses enfants ${ }^{21}$ " (jammert, eines seiner Kinder verzehrt zu haben). Toute chose, tout aspect deviennent alors les instruments d'un mensonge et d'un danger: le pain suisse (Bürli) semble à Warburg si suspect qu'il réclame des galettes azymes; la fleur devient menaçante, le thé n'est qu'une décoction de sang humain ou le philtre imaginé par quelque "clique antisémite "; le poisson contient son propre fils et, depuis son assiette, l'implore en ces termes : "Père, ne me mange pas » (Vater, du wirst mich doch nicht essen); son gâteau d'anniversaire, le 13 juin 1922, est «fait avec quelque chose de bien pire que le sang humain ${ }^{22}$.» 

situation recelait un danger. Comme à la guerre, toute information était faussée par le mensonge et la propagande (l'un de ses grands thèmes de recherche entre 1914 et 1918). Ainsi, «le beurre est de la graisse de mouche, le pain n'est pas du pain »; les épreuves de son article sur Luther sont " fausses »; « le chou frisé est la cervelle de son frère, les pommes de terre sont les têtes de ses enfants, la viande est la chair des membres de sa famille »; les articles de journaux sur la nomination de son frère au rang de docteur honoris causa sont des mensonges; et Warburg de scander toutes ces suspicions en imprécations, en galimatias et en néologismes de toutes sortes ${ }^{23}$. Ce qui ne l'empêche pas de consigner au jour le jour, en bon superstes, tous les éléments de sa psychomachie (c'est le matériel, encore inédit, des carnets de Kreuzlingen conservés au Warburg Institute de Londres). Aussi se montre-t-il «très agité lorsqu'on lui retire le gros paquet de lettres qu'il avait jusqu'alors gardées avec lui, parmi lesquelles certaines datent du temps où il était chez le docteur Lienau, ainsi qu'un journal, entièrement déchiré, datant de cette époque également ${ }^{24}$. "

L'interminable guerre psychique de Warburg après 1918 fut une guerre pathologique, sans doute : elle répondait à son destin personnel ou à sa petite histoire, par exemple lorsqu'il fit de sa relation amoureuse avec la gouvernante anglaise de la famille un motif délirant de culpabilité politique sur son propre rôle dans la défaite allemande ${ }^{25}$. Mais cette guerre était, aussi, en phase - sismographique - avec la grande histoire, par exemple lorsque Binswanger raconte, en 1922, que son patient se montre «très perturbé par la mort de Rathenau [et] croit (hält) que son frère est très en danger ${ }^{26}$.» En employant le verbe halten, qui d'abord signifie «tenir » et "maintenir ", Binswanger évitait subtilement de suggérer que son patient était possédé par une simple croyance délirante : il aura peut-être su que le groupuscule politique qui avait assassiné Walter Rathenau, le 24 juin 1922, s'apprêtait en effet à tuer Max Warburg quelques jours plus $\operatorname{tard}^{27}$.

On comprend alors que l'auteur de Mnémosyne fut en effet ce qu'il admirait chez Nietzsche : un « sismographe très sensible » et un «voyant » du type nabi. Capable, à ce titre, de souffrir follement, de voler en éclats. Mais demeurant, dans ce pathos même, à l'écoute des mouvements impersonnels et souterrains, des basses continues de l'histoire objective. Quand Warburg «voit » dans le jardin de Kreuzlingen des « caisses remplies de chair humaine » ou des terrains aménagés "afin d'enterrer vivants des hommes ${ }^{28}$ ", il ne fait que déplacer - et rapprocher de lui jusqu'à l'incorporation - une réalité historique partout visible lors de la Grande Guerre; lorsqu'il imagine sa bibliothèque en flammes ${ }^{29}$, il ne fait que présager le destin que les nazis hambourgeois voulurent précisément réserver, en 1933, à la Kulturwissenschaftliche Bibliothek Warburg ; lorsqu'il s'effraie de "colonie pénitentiaire ${ }^{30}$ " (Verbrecherkolonie), il se situe quelque part entre une fiction de Strafkolonie à la Kafka et la future réalité des camps de concentration nazis à laquelle il est difficile de ne pas songer lorsque, tissant le motif récurrent de la haine antisémite, Warburg en vient à croire que « le vieux bois que l'on brûlait était les membres de sa famille ${ }^{31}$.» Quand il parle d'une "politique de catastrophe " (Katastrophenpolitik), on ne sait plus trop s'il accuse les médecins autour de lui ou bien les dirigeants de l'Europe tout entière ${ }^{32}$.

L'histoire clinique d'Aby Warburg ne nous intéresserait pas si elle n'était qu'un épisode purement subjectif, un simple défaut dans son « espace de pensée ». Mais elle est bien plus que cela. Elle se développe d'une façon dialectique, toujours sur deux plans 
hétérogènes, conflictuels, qui ne cessent pourtant de se recroiser : le non-savoir et le savoir, le pathos et le logos, l'histoire personnelle et l'histoire tour court. C'est ainsi qu'il faut comprendre la grande "psychomachie» de Warburg. Ne nous étonnons pas qu'il « souffrit longtemps du sentiment d'avoir une tête de Janus [et] affirmait en éprouver une sensation très nette ${ }^{33}$. » À Kreuzlingen, son état aura le plus souvent été qualifié d'« oscillatoire » (häufiger schwankt) par Binswanger : «Pendant des heures, il peut être aimable, calme, sympathique, avoir une conversation brillante sur des thèmes scientifiques, faire de l'esprit avec à-propos ; et soudain, tout se renverse, il entre dans un état d'agitation terrible, d'une intensité qu'on n'avait plus observée depuis longtemps, il use des expressions les plus grossières et devient agressif ${ }^{34}$.» Tour à tour il argumente et invective, raisonne et vocifère, travaille et se lamente, classe ses papiers et jette tout par-dessus bord, s'apaise et se ré-angoisse, se met à crier pour finir sur un jeu de mots extraordinairement spirituel ${ }^{35}$. Son entourage aura longtemps cru que cette guerre n'aurait pas de fin.

17 Et cependant, comme on le sait, Aby Warburg aura fini par quitter Kreuzlingen, sa «colonie pénitentiaire » et par recouvrer la raison, retrouver sa chère bibliothèque pour se lancer dans l'ultime grand projet de sa vie, l'atlas d'images Mnémosyne. Mais il en est de ce «miracle » et de cette " guérison » individuelle comme de la guerre et de la «psychomachie» culturelle où Warburg s'était débattu avec tant d'énergies douloureuses: leur temporalité n'est pas réductible à celle d'épisodes historiques aisément situables. Ce "miracle» et cette "guérison" ont subi, de la part des historiens, nombre de simplifications, voire de mythifications biographiques et méthodologiques. D'un côté, Ernst Gombrich n'a vu dans Mnémosyne qu'une « solution à l'impasse » où demeurait Warburg à son retour de la folie ${ }^{36}:$ à celui qui ne savait plus quoi dire, il ne restait, en somme, qu'à reclasser les images de sa photothèque. Façon de méconnaitre la teneur heuristique, ouverte et théoriquement si novatrice, du projet de Mnémosyne. D'un autre côté, l'atlas d'images est apparu comme l'incarnation même du «miracle de guérison »: une manière de sauvetage qu'il faudrait porter au crédit de Fritz Saxl, puisque celui-ci, accueillant son maître à peine rentré de l'asile, organisa une "fête» en disposant, dans la salle de lecture de Hambourg, quelques panneaux qui résumaient en images les thèmes fondamentaux de la recherche warburgienne ${ }^{37}$.

Il est sans doute nécessaire d'établir une chronologie de Mnémosyne, en repérant par exemple les mentions du projet dans ce document étonnant qu'est le Tagebuch, le « journal » de la Kulturwissenschaftliche Bibliothek Warburg, tenu à plusieurs mains entre 1926 et $1929^{38}$. Et, bien sûr, de distinguer les trois principales versions que connut l'élaboration du projet, demeuré inachevé à la mort de Warburg ${ }^{39}$. Mais je voudrais poser ici un autre genre de questions : quel espace de pensée l'atlas Mnémosyne inventet-il exactement? Quel destin réserve-t-il à l'inquiétude foncière de la méthode warburgienne et au déséquilibre profond qui en résulta entre 1918 et 1924 ? Le déséquilibre, chez Warburg, fut tellement lié à l'inquiétude - c'est-à-dire à la méthode elle-même - qu'on est en droit de douter du caractère unilatéralement " salvateur » de Mnémosyne dans l'économie de sa pensée. L'atlas Mnémosyne ne signe pas une «sortie » de l'inquiétude ou une réassurance tranquille de la recherche «scientifique ». Bien au contraire, il constitue la géniale reformulation de cette inquiétude même, sa recomposition pratique et théorique, sa reconduction sous de nouvelles formes, son remontage. Il porte en lui, vivace, cette " connaissance du souffrant " qu'incarne bien le titan Atlas (au plan mythologique) et dont Nietzsche aura fait la pointe de toute pensée 
(au plan philosophique). Quelles seraient, pour finir, les leçons politiques d'une telle inquiétude devant l'histoire vouée au déséquilibre de toute chronique?

On sait qu'un moment fondamental, dans la «psychomachie » de Warburg pour revenir $d u$ désastre où la Grande Guerre l'avait plongé, fut la conférence prononcée à Kreuzlingen en 1923 sur le « rituel du serpent » des Indiens Hopi ${ }^{40}$. Les notes cliniques de Ludwig Binswanger, témoin de l'événement et son habile accompagnateur, nous apparaissent ici fort précieuses dans la mesure où le psychiatre, comme on sait, fut un véritable théoricien $\mathrm{du}$ " savoir pathique » et un observateur attentif du "style d'être " de chacun de ses patients ${ }^{41}$. Le 10 mars 1923, il note chez Warburg un état "agité, furieux, violent "; le 12 mars, Fritz Saxl arrive de Hambourg pour aider le savant dans la préparation de sa conférence; le 18 mars, « fin de la cure d'opium. Elle n'a apporté aucun apaisement. Le patient était aussi mal qu'auparavant " ; mais, grâce à la présence de son assistant, Warburg devient "plus calme [et] travaille assez régulièrement à sa conférence »; il n'en demeure pas moins l'homme à la «tête de Janus " puisque, «l'élaboration de son travail avanç[ant] bien [...], l'état d'esprit fondamentalement délirant demeure ${ }^{42}$.»

La conférence eut finalement lieu le 21 avril 1923. Binswanger en aura fait pour luimême un résumé plutôt sommaire, préférant noter ce qui le frappait dans le style ou la présentation même du discours tenu : la « surprenante maitrise intellectuelle " alliée à un «dynamisme » de l'argumentation, cependant déséquilibrée par le timbre cassé de la voix de l'orateur; la "grande quantité de connaissances [présentées] d'une manière un peu désordonnée "; et, surtout, le fait que "le patient s'est beaucoup soucié de la mise en scène des images " (Inszenierung der Lichtbilder), en sorte que "la conférence elle-même était plus une causerie rattachée au matériau photographique ${ }^{43}$ " (Photomaterial). Entre deux crises - ou entre d'innombrables émissions de cris -, Aby Warburg trouvait donc dans une certaine présentation visuelle la possible table d'orientation de sa pensée, ce que Binswanger note également à un moment où il observe justement l'appréhension psychique de l'espace chez son patient :

« 4 juin [1924]. Transfert à la Villa Maria. [Warburg] s'était peu à peu habitué à l'idée de ce déménagement, mais [...] le plus difficile est pour lui de s'habituer aux nouvelles pièces, particulièrement à la salle de bains. [...] Il lui faut désormais mettre la présente salle de bains en accord avec celles d'Iéna et de Parkhaus, ce qui lui pose les pires problèmes. Il est déjà très difficile de reconstruire en mémoire (in der Erinnerung zu rekonstruieren) la salle de bains de Parkhaus. Le patient est également très troublé, dans les autres lieux, par la nouvelle organisation de l'espace ; il est proprement désorienté (eigentlich desorientiert), car, par exemple, l'axe de la table est disposé différemment par rapport à la fenêtre, si bien que, de la fenêtre, il doit faire d'autres mouvements pour rejoindre la table, etc. Pose sur la table des objets qui ont une résonance affective, des livres, des images, parce qu'ils facilitent son orientation (die Orientierung erleichtert). Ainsi, quand il lit, il regarde en cachette ces objets qui lui apportent un certain apaisement. Pour cette raison, on ne peut lui faire passer son habitude de transporter partout ses affaires ${ }^{44}$. »

21 On comprend alors que, dans cette psychopathologie de l'espace visuel - dont Binswanger aura, quelques années plus tard, tenté de ressaisir toutes les données sur le plan phénoménologique ${ }^{45}-$, Aby Warburg ne pouvait accorder son attention à une chose qu'en la reliant à d'autres choses affines pour former une constellation dans laquelle il pouvait retrouver une orientation pour sa pensée. D'où cette « habitude de transporter partout ses affaires", qui, une fois de plus, apparente l'auteur de Mnémosyne au titan Atlas, à la figure du Juif errant ou à celle du chiffonnier benjaminien 
- mais un chiffonnier accumulant manuscrits, fiches et photographies pour tenter de recueillir le morcellement du monde par plans de pensée ou par planches, par tables d'orientation interposés.

Plans de la pensée ou tables d'orientation : c'est donc cela qu'il fallait à Warburg pour ne pas sombrer tout à fait dans les disparates du monde, les caprices de l'imagination ou les désastres de l'histoire. C'est bien cela qu'il lui fallait pour engager victorieusement sa psychomachie des astra et des monstra. Les tables d'orientation comme déjà les foies divinatoires babyloniens, la carte céleste de l'Atlas Farnèse ou, bientôt, les planches mêmes de l'atlas Mnémosyne - auront été pour Warburg ce que son inquiétude elle-même exigeait, méthodiquement, pour ne pas sombrer tout à fait dans le chaos. «Je ne vois que chaos devant moi » (ich sehe nur Chaos vor Augen), écrivaitil le 7 avril 1924, quelques jours avant une visite d'Ernst Cassirer qui le « réorienta » et grâce à laquelle il put sentir renaître en lui quelque chose comme une "puissance de libération (Befreiung) à l'égard du trouble psychique ${ }^{46}$.

Une table d'orientation, au sens divinatoire du terme, suppose la circulation constante des espaces maléfiques et bénéfiques, donc des moments mélancoliques (chutes dans le temps) et maniaques (triomphes sur le destin) : pars hostilis d'un côté, pars familiaris de l'autre. Il s'agissait pour Warburg, entre 1918 et 1924, de faire retourner le disque ou la «table » de la première vers la seconde, fût-ce dans un mouvement où il fallait en repasser, fatalement, par les mauvaises cases du destin. Pars hostilis: ce sont, par exemple, les interrogations paranoïaques de Warburg lorsqu'il demande, arrivant tout juste à Kreuzlingen, de quoi il est accusée ${ }^{47}$. Pars familiaris: ce sera, de retour à Hambourg, l'interrogation elle-même pensée comme l'éthos par excellence du chercheur:

«Voilà bien une question d'éthos scientifique (eine Frage des wissenschaftlichen Ethos): a-t-on l'ambition de susciter de la part des étudiants le point d'exclamation de l'admiration ou le point d'interrogation de la modestie ${ }^{48}$ (Fragezeichen der Selbstbescheidung)?»

Un autre exemple de cette désorientation (funeste) et de la réorientation (bénéfique) du Denkraum warburgien concerne, justement, la fonction mémorative des images. Warburg, assistant à une conférence de Binswanger à Kreuzlingen, prend quelques notes furtives qui dérivent très vite vers ses propres enjeux de «psycho-historien ». Il écrit : "Image et signe " (Bild und Zeichen) puis, aussitôt: "Sélection phobique de la fonction de la mémoire en images " (phobische Auslese der Funktion des Bildgedächtnisses), motif qui sera encore, dans le texte introductif de Mnémosyne, en 1929, le point focal de toute sa réflexion sur la polarisation - terreur et attrait, monstra et astra - des images ${ }^{49}$. Les fragments autobiographiques écrits par Warburg à Kreuzlingen portent tous témoignage de cette capacité des images mémorielles à fonctionner alternativement comme pars familiaris et pars hostilis: grâce à Darwin puis à Hegel, Warburg aura découvert le principe fondamental d'une "immanence de la loi» (Immanenz des Gesetzes) qui fut aussi le moteur irrationnel de toutes ses " hallucinations phobiques", de ses "images démoniaques » et de ce qu'il appelle, à un moment, ses « esprits » ou « dames pst-pst ${ }^{50}$ » (Pst-Pst Damen). En Warburg, donc, le savant avait bien compris la double fonction culturelle des images - astra et monstra - sans qu'il lui fût jamais garanti, comme patient, d'échapper à cette oscillation même dont il avait, depuis l'enfance, éprouvé toute la puissance :

"J'ai conservé de cette période [une fièvre typhoïde contractée à l'âge de six ans]

les images qui me venaient dans les hallucinations fébriles, et par leur netteté elles 
m'impressionnent comme autrefois. [...] De ce temps me viennent la peur suscitée par l'incohérence et la force disproportionnée des souvenirs visuels (unproportioniert zusammenhangslose Bildererinnerungen) ou des sensations olfactives et auditives, l'angoisse qui fait naître le chaos, et la tentative d'instituer un ordre intellectuel dans ce chaos (intellektuell Ordnung in dieses Chaos zu bringen) - cette tragique tentative infantile (tragische Kindheitsversuch) de l'homme pensant commença donc très tôt pour moi ${ }^{51}$.»

La mémoire serait donc tout à la fois ce qui fixait Warburg à la part funeste des irrémissibles monstra et ce qui lui permettait de viser la part bénéfique des astra dans une "tentative d'autolibération par le souvenir (Selbstbefreiungs-Versuch durch die Erinnerung) de [ses] tentatives d'éclaircissement (Aufklärungsversuche) en matière de psychologie de la Renaissance » et d'histoire culturelle en général ${ }^{52}$. C'est en ce sens que le séjour à Kreuzlingen ne fut pas seulement une parenthèse dans la folie, mais bien une construction ou une reconstruction, une réorientation de la folle puissance des images sur le destin des hommes éprouvée, comme jamais auparavant, dans le travail mené à Hambourg entre 1914 et $1918^{53}$. La correspondance échangée entre Ludwig Binswanger et Aby Warburg après le retour de celui-ci à Hambourg ${ }^{54}$ - et jusqu'à sa mort - nous permet de mieux saisir encore ce travail de réorientation dont le résultat n'est autre que l'atlas Mnémosyne lui-même, ce grand recueil de tables d'orientation: des tables ou des " planches » pour refaire ce que la guerre avait défait et pour comprendre la grande "psychomachie» occidentale selon le jeu destinal de la pars hostilis des images et de leur capacité à venir, malgré tout, jouer pleinement leur rôle dans la pars familiaris de notre pensée.

Binswanger commente d'abord avec justesse le sens même de l'anamnèse intellectuelle de Warburg comme un prolongement de son anamnèse "pathique» conduite à Kreuzlingen : «Ce que vous me dites des développements de votre travail m'a beaucoup intéressé ", écrit-il à l'historien le 28 décembre 1925. Et il précise aussitôt: "Cette manière de tracer un arc de cercle en arrière (dieses Bogenschlagen nach rückwärts) représente aussi une tension vers l'avant ${ }^{55}$ (ein Aufstreben nach vorwärts).» Warburg confirmera en ces termes : " J'ai énormément à faire, ma productivité intellectuelle me donne un grand désir d'entreprendre (unternehmungslustig), au point que ma très chère psyché commence à retisser fidèlement les fils des dernières idées que j'avais avant la

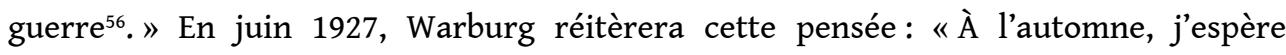
retourner en Italie pour achever une série d'études que la guerre avait interrompues ${ }^{57}$.»

Mais ces études auront, nous le savons, pris un tour inattendu quoique prévisible. Une orientation ou, plutôt, une présentation nouvelles : c'est, en 1926, une "exposition destinée au colloque des orientalistes allemands [...] en relation avec la troisième édition de Sternglaube und Sterndeutung de Boll » étendue à un projet d'atlas qui devrait "montrer la migration des symboles astraux" et nécessite pour cela toute une logistique photographique - «la Photoclark du Dr. Jantsch, d'Uberlingen, [qui] permet d'obtenir en peu de temps une quantité énorme d'images, sans avoir besoin de négatifs de verre" -, tout cela pour rendre visibles certaines "considérations sur la psychologie des images ${ }^{58} »$. Ce travail n'est autre que Mnémosyne, dont Warburg confiera bientôt à Binswanger qu'il «commence à repousser ses limites initiales » et rend son achèvement problématique, le psychiatre répondant alors - marque d'admiration ou d'inquiétude? - que ce nouveau projet de Warburg ressemble à un « travail monstrueux ${ }^{59}$ » (eine horrende Arbeit!). 
Horrend: " horrifique ", "terrifiant " mais, aussi, "énorme », " considérable ", «formidable ». Pourquoi si terrifiant? Parce que le projet inhérent à Mnémosyne n'est autre qu'une "histoire de fantômes pour grandes personnes» commencée dans l'horreur de la Grande Guerre, passée de l'inquiétude au déséquilibre puis à la folie de son auteur, et qui devait se finir par le cauchemar annoncé de la victoire des fascismes en Europe (fig. 43-44). Pourquoi si formidable? Parce que Mnémosyne aura eu pour ambition de remonter un monde démonté par les désastres de l'histoire, d'en renouer les fils mémoriels par-delà ses épisodes, d'en renouveler la cosmographie intellectuelle, comme si la sphère portée par le titan mythologique, dans l'Atlas Farnèse, détruite par les temps modernes, devait être entièrement recomposée, redessinée à nouveaux frais par ce voyant du temps que fut Aby Warburg ${ }^{60}$.

Ce n'est pas un hasard si le vocabulaire employé par l'historien des images, au retour de Kreuzlingen, suggérait une réponse de la pensée à cette « dislocation du monde » que représentait la guerre à ses yeux. Que sont donc les armes de la pensée contre celles de la lutte militaire que les hommes ne cessent de mener contre eux-mêmes? Warburg parla souvent de sa bibliothèque - dont l'entrée s'ornait d'une inscription en grec, MNEMOSYNH, pour Mnémosyne - comme d'une «citadelle de livres ${ }^{61}$ " (Büchertrutzkasten). En 1927, dans un texte autobiographique où le traumatisme de la Grande Guerre affleurait encore, il voulut jouer sur les deux mots «arsenal » et «laboratoire ${ }^{62}$ ». Il y réitéra son idée d'une « citadelle » pour la pensée. Mais ce n'était pas une tour d'ivoire refermée sur ses propres triomphes d'érudition, comme l'aurait voulu un savant positiviste ou idéaliste: plutôt un dispositif expérimental faisant du Denkraum warburgien quelque chose comme un laboratoire capable de s'inventer, en permanence, des appareils pour voir le temps à l'œuvre dans les paroles, les images et les gestes humains.

\section{BIBLIOGRAPHIE}

Bauerle D., 1988. Gespenstergeschichten für ganz Erwachsene. Ein Kommentar zu Aby Warburgs Bilderatlas Mnemosyne, Münster, Lit, 1988.

Bender C., Hensel T. et Schüttpelz E. (dir.), 2007. Schlangenritual. Der Transfer der Wissensformen vom Tsu'ti'kive der Hopi bis zu Aby Warburgs Kreuzlinger Vortrag, Berlin, Akademie Verlag, 2007.

Binswanger L., 1921-1924. « Histoire clinique [d'Aby Warburg] », trad. M. Renouard et M. Rueff, La Guérison infinie. Histoire clinique d'Aby Warburg, éd. D. Stimilli, Paris, Payot \& Rivages, 2007, p. 53-180.

Binswanger L., 1933. Le Problème de l'espace en psychopathologie, trad. C. Gros-Azorin, Toulouse, Presses universitaires du Mirail, 1998.

Binswanger L. et Warburg A., 1924-1929. « Correspondance », trad. M. Renouard et M. Rueff, La Guérison infinie. Histoire clinique d'Aby Warburg, éd. D. Stimilli, Paris, Payot \& Rivages, 2007, p. 227-298.

Images Re-vues, Hors-série 4 | 2013 
Bologna C., 2004. « Mnemosyne : il "teatro della memoria" di Aby Warburg », Lo sguardo di Giano. Aby Warburg fra tempo e memoria, dir. C. Cieri Via et P. Montani, Turin-Racconigi, Nino Aragno Editore, 2004, p. 277-304.

Careri G., 2003. « Aby Warburg : rituel, Patosformel et forme intermédiaire », L'Homme, $\mathrm{n}^{\circ} 165$, 2003, p. 41-76.

Centanni M. et Ferlenga A. (dir.), 2004. Mnemosyne. L'atlante di Aby Warburg, Venise, EngrammaFondazione Ugo e Olga Levi, 2004.

Centanni M. et Mazzucco K., 2002. «Letture da Mnemosyne », Introduzione ad Aby Warburge all'Atlante della memoria, dir. M. Centanni, Milan, Bruno Mondadori, 2002, p. 166-238.

Cestelli Guidi B., 2003. « La collection pueblo d'Aby Warburg, 1895-1896. Culture matérielle et origines de l'image ", trad. D. H. Bodart, dans A. Warburg, Le Rituel du serpent. Récit d'un voyage en pays pueblo, trad. S. Muller, Paris, Macula, 2003, p. 163-192.

Cestelli Guidi B., 2004. « “Trattate con cura i miei libri e le mie rarità”. Aby Warburg collezionista ", Lo sguardo di Giano. Aby Warburg fra tempo e memoria, dir. C. Cieri Via et P. Montani, Turin-Racconigi, Nino Aragno Editore, 2004, p. 523-568.

Cestelli Guidi B. et Mann N. (dir.), 1998. Photographs at the Frontier : Aby Warburg in America, 1895-1896, Londres, The Warburg Institute-Merrell Holberton, 1998.

Chernow R., 1993. The Warburgs. The Twentieth-Century Odyssey of a Remarkable Jewish Family, New York, Vintage Books, 1993.

Didi-Huberman G., 2002. L'Image survivante. Histoire de l'art et temps des fantômes selon Aby Warburg, Paris, Les Éditions de Minuit, 2002.

Diers M., 1979. « Kreuzlinger Passion », Kritische Berichte, VII, 1979, p. 5-14.

Fédida P., 1970. « Binswanger et l'impossibilité de conclure », préface à L. Binswanger, Analyse existentielle, psychiatrie clinique et psychanalyse. Discours, parcours, et Freud, Paris, Gallimard, 1970, p. 7-37.

Forster K. W., 1991. « Die Hamburg-Amerika-Linie, oder : Warburgs Kulturwissenschaft zwischen den Kontinenten ", Aby Warburg. Akten des internationalen Symposions Hamburg 1990, dir. H. Bredekamp, M. Diers et C. Schoell-Glass, Weinheim, VCH-Acta Humaniora, p. 11-37.

Füssel S. (dir.), 1979. Mnemosyne, Göttingen, Gratia Verlag, 1979.

García Lorca F., 1930. « Théorie et jeu du duende », trad. A. Belamich, Euvres complètes, I, Paris, Gallimard, 1981 (éd. 1986), p. 919-931.

Gockel B., 2007. « Krieg - Krankheit - Kulturwissenschaft. Warburgs Schizophrenie als Forschungsinstrument und das Ideal moderner Primitivität ", Kasten 117. Aby Warburg und der Aberglaube im Ersten Weltkrieg, dir. G. Korff, Tübingen, Tübinger Vereinigung für Volkskunde, 2007, p. 117-134.

Gombrich E. H., 1970. Aby Warburg. An Intellectual Biography, Londres, The Warburg Institute (2 édition, Chicago-Oxford, The University of Chicago Press-Phaidon, 1986).

Grazioli M., 2006. Saxl interprete di Mnemosyne. Individuazione di un apparato critico-descrittivo dell'Atlante della memoria di Aby Warburg nei saggi del suo più stretto collaboratore, Thèse, Bologne, Alma Mater Studiorum-Università di Bologna, 2006. 
Huisstede P. van, 1995. « Der Mnemosyne-Atlas. Ein Laboratorium der Bildergeschichte », Aby M. Warburg. «Ekstatische Nymphe... trauernder Flussgott ». Portrait eines Gelehrten, dir. R. Galitz et B. Reimers, Hambourg, Dölling und Galitz, 1995, p. 130-171.

Janshen F., 1993. « Spurenlesen. Um Aby Warburgs "Schlangenritual” », Denkräume. Zwischen Kunst und Wissenschaft, dir. S. Baumgart et al., Berlin, Dietrich Reimer, 1993, p. 87-112.

Koerner J. L., 1997. «Introduction », trad. S. Muller et P. Guiton, dans A. Warburg, Le Rituel du serpent. Récit d'un voyage en pays pueblo, Paris, Macula, 2003, p. 9-54.

Königseder K., 1995. « Aby Warburg im “Bellevue” ", Aby M. Warburg. «Ekstatische Nymphe... trauernder Flussgott ». Portrait eines Gelehrten, dir. R. Galitz et B. Reimers, Hambourg, Dölling und Galitz, 1995, p. 74-98.

Koos M., Pichler W., Rappl W. et Swoboda G. (dir.), 1994. Begleitmaterial zur Ausstellung « Aby Warburg. Mnemosyne », Hambourg, Dölling und Galitz, 1994.

Lienau A., 1918-1919. « Krankengeschichte Hamburg 2. November 1918-17. Juli 1919 », Die unendliche Heilung. Aby Warburgs Krankengeschichte, éd. C. Marazia et D. Stimilli, Zürich-Berlin, Diaphanes, 2007, p. 213-220.

Mann N., 2002. « Mnemosyne : "Dalla parola all'immagine". Prefazione all'edizione italiana », dans A. Warburg, Mnemosyne. L'atlante delle immagini, trad. dirigée par M. Ghelardi, Turin-Racconigi, Nino Aragno Editore, 2002, p. VII-XI.

Mazzucco K., 2002a. " Genesi di un'opera "non finibile” ", Introduzione ad Aby Warburg e all'Atlante della memoria, dir. M. Centanni, Milan, Bruno Mondadori, 2002, p. 55-84.

Mazzucco K., 2002b. «I pannelli di Mnemosyne », Introduzione ad Aby Warburg e all'Atlante della memoria, dir. M. Centanni, Milan, Bruno Mondadori, 2002, p. 85-165.

McEwan D., 2004. « Wanderstrassen der Kultur ». Die Aby Warburg - Fritz Saxl Korrespondenz, 1920 bis 1929, Munich-Hambourg, Dölling und Galitz Verlag, 2004.

Michaud P.-A., 1998. Aby Warburg et l'image en mouvement, Paris, Macula, 1998.

Naber C., 1988. « Pompeji in Neu-Mexico. Aby Warburgs amerikanische Reise », Freibeuter, XXXVIII, 1988, p. 88-97.

Nietzsche F., 1881. Aurore. Pensées sur les préjugés moraux, trad. J. Hervier, CEuvres philosophiques complètes, IV, éd. G. Colli et M. Montinari, Paris, Gallimard, 1980.

Raulff U., 1988. « Nachwort », dans A. M. Warburg, Schlangenritual. Ein Reisebericht, Berlin, Verlag Klaus Wagenbach, 1988 (éd. 1996), p. 59-95.

Raulff U., 1991. « Zur Korrespondenz Ludwig Binswanger-Aby Warburg im Universitätsarchiv Tübingen », Aby Warburg. Akten des internationalen Symposions Hamburg 1990, dir. H. Bredekamp, M. Diers et C. Schoell-Glass, Weinheim, VCH-Acta Humaniora, p. 55-70.

Raulff U., 1998. «The Seven Skins of the Snake. Oraibi, Kreuzlingen and back : Stations on a Journey into Light », trad. D. McEwan, dans B. Cestelli Guidi et N. Mann (dir.), Photographs at the Frontier : Aby Warburg in America, 1895-1896, Londres, The Warburg Institute-Merrell Holberton, 1998, p. 64-74.

Raulff U., 2003. Wilde Energien. Vier Versuche zu Aby Warburg, Göttingen, Wallstein Verlag, 2003. Saxl F., 1922-1923. « Notes sur Kreuzlingen ", trad. M. Renouard et M. Rueff, La Guérison infinie. Histoire clinique d'Aby Warburg, éd. D. Stimilli, Paris, Payot \& Rivages, 2007, p. 217-225. 
Saxl F., 1929-1930. « Le voyage de Warburg au Nouveau-Mexique », trad. S. Muller et P. Guiton, dans A. Warburg, Le Rituel du serpent. Récit d'un voyage en pays pueblo, Paris, Macula, 2003, p. 149-161.

Schüttpelz E., 2007. « Das Schlangenritual der Hopi und Aby Warburgs Kreuzlinger Vortrag », Schlangenritual. Der Transfer der Wissensformen vom Tsu'ti'kive der Hopi bis zu Aby Warburgs Kreuzlinger Vortrag, dir. C. Bender, T. Hensel et E. Schüttpelz, Berlin, Akademie Verlag, 2007, p. 187-216.

Severi C., 2003. « Warburg anthropologue, ou le déchiffrement d'une utopie. De la biologie des images à l'anthropologie de la mémoire ", L'Homme, n 165, 2003, p. 77-128.

Severi C., 2004. Il percorso e la voce. Un' antropologia della memoria, Turin, Einaudi, 2004.

Spinelli I. et Venuti R. (dir.), 1998. Mnemosyne. L'Atlante della memoria di Aby Warburg, Rome, Artemide Edizioni, 1998.

Stimilli D., 2005. « La teinture de Warburg », trad. M. Renouard et M. Rueff, La Guérison infinie. Histoire clinique d'Aby Warburg, éd. D. Stimilli, Paris, Payot \& Rivages, 2007, p. 7-52.

Tavani E., 2004. « L'Atlante Farnese nel Bilderatlas di Aby Warburg », Il classico violato. Per un museo letterario del '900, dir. R. Ascarelli, Rome, Artemide Edizioni, 2004, p. 121-143.

Warburg A., 1921-1924. « Lettres et fragments autobiographiques », trad. M. Renouard et M. Rueff, La Guérison infinie. Histoire clinique d'Aby Warburg, éd. D. Stimilli, Paris, Payot \& Rivages, 2007, p. 181-215.

Warburg A., 1923. Le Rituel du serpent. Récit d'un voyage en pays pueblo, trad. S. Muller, Paris, Macula, 2003.

Warburg A., 1926-1929. Tagebuch der Kulturwissesnchaftlichen Bibliothek Warburg, mit Einträgen von Gertrud Bing und Fritz Saxl. Gesammelte Schriften, VII, éd. K. Michels et C. Schoell-Glass, Berlin, Akademie Verlag, 2001.

Warburg A., 1927a. Vom Arsenal zum Laboratorium, Londres, Warburg Institute Archive, V, 2.3.1.1. Trad. M. Ghelardi, « Da arsenale a laboratorio », Belfagor, LVI, 2001, n 2, p. 175-183.

Warburg A., 1927b. « Texte de clôture du séminaire sur Burckhardt », trad. D. Meur, Les Cahiers du Musée national d'Art moderne, $n^{\circ}$ 68, 1999, p. 21-23.

Warburg A., 1929. « Mnémosyne (introduction)», trad. P. Rusch, Trafic, n 9, 1994, p. 38-44.

Warnke M., 2000. « Editorische Vorbemerkungen », dans A. Warburg, Der Bilderatlas Mnemosyne. Gesammelte Schriften, II-1, éd. M. Warnke et C. Brink, Berlin, Akademie Verlag, 2000 ( 2 ème éd. revue, 2003), p. VII-X.

\section{NOTES}

1. A. Lienau, 1918-1919, p. 213-214.

2. Cité par L. Binswanger, 1921-1924, p. 61-62.

3. Ibid., p. 89-90.

4. Cf. M. Diers, 1979, p. 5-14. K. Königseder, 1995, p. 74-98. G. Didi-Huberman, 2002, p. 363-413.

5. A. Warburg, 1923, p. 55-133. D. Stimilli, 2005, p. 7-52.

6. Cité par D. Stimilli, 2005, p. 9.

7. Ibid., p. 15.

8. Ibid., p. 19-25. 
9. A. Warburg, 1921-1924, p. 187.

10. A. Warburg, 1927b, p. 21.

11. Ibid., p. 21-22.

12. F. Nietzsche, 1881, p. 94.

13. F. García Lorca, 1930, p. 919-931.

14. Cité par L. Binswanger, 1921-1924, p. 61 (cf. également p. 86).

15. Ibid., p. 120.

16. Ibid., p. 97.

17. Ibid., p. 100.

18. Ibid., p. 180.

19. F. Saxl, 1922-1923, p. 222.

20. L. Binswanger, 1921-1924, p. 77.

21. Ibid., p. 145-146.

22. Ibid., p. 91, 93, 96-97, 120 et 129.

23. Ibid., p. $74-77$ et 106-110.

24. Ibid., p. 84 .

25. Ibid., p. 88.

26. Ibid., p. 129.

27. Cf. R. Chernow, 1993, p. 228-229.

28. L. Binswanger, 1921-1924, p. 99 et 101.

29. Ibid., p. 105.

30. Ibid., p. 133.

31. Ibid., p. 137.

32. Ibid., p. 158.

33. Ibid., p. 85.

34. Ibid., p. 138.

35. Ibid., p. 68, 82-85, 138-143, 173-180.

36. E. H. Gombrich, 1970, p. 285.

37. Cf. N. Mann, 2002, p. VII-VIII. D. McEwan, 2004, p. 110-112. M. Grazioli, 2006.

38. A. Warburg, 1926-1929, p. 126-127, 147-148, 167-170, 245-255, 326-338, 434-437, 543-555, etc.

39. M. Warnke, 2000, p. VII-X. Cf. S. Füssel (dir.), 1979. D. Bauerle, 1988, p. 65-142. M. Koos, W. Pichler, W. Rappl et G. Swoboda (dir.), 1994. P. van Huisstede, 1995, p. 130-171. T. Spinelli et R. Venuti (dir.), 1998. M. Centanni et K. Mazzucco, 2002, p. 166-238. K. Mazzucco, 2002a, p. 55-84. Id., 2002b, p. 85-165. M. Centanni et A. Ferlenga (dir.), 2004.

40. A. Warburg, 1923, p. 55-133. Cf. F. Saxl, 1929-1930, p. 149-161. C. Naber, 1988, p. 88-97. U. Raulff, 1988, p. 59-95. K. W. Forster, 1991, p. 11-37. F. Janshen, 1993, p. 87-112. J. L. Koerner, 1997, p. 9-54. B. Cestelli Guidi et N. Mann (dir.), 1998. P.-A. Michaud, 1998, p. 169-223. U. Raulff, 1998, p. 64-74. G. Careri, 2003, p. 41-76. B. Cestelli Guidi, 2003, p. 163-192. U. Raulff, 2003. C. Severi, 2003, p. 77-128. Id., 2004, p. 21-86. B. Cestelli Guidi, 2004, p. 523-568. C. Bender, T. Hensel et E. Schüttpelz (dir.), 2007. E. Schüttpelz, 2007, p. 187-216.

41. Cf. P. Fédida, 1970, p. 7-37.

42. L. Binswanger, 1921-1924, p. 154-155.

43. Ibid., p. 156-157.

44. Ibid., p. 176-177.

45. Id., 1933.

46. A. Warburg, 1921-1924, p. 206 et 214.

47. Ibid., p. 183-187.

48. L. Binswanger et A. Warburg, 1924-1929, p. 253 (lettre du 23 décembre 1925).

49. A. Warburg, 1921-1924, p. 199. Cf. id., 1929, p. 38-44.

50. Id., 1921-1924, p. 195-198 et 203. 
51. Ibid., p. 189-190.

52. Ibid., p. 204.

53. Cf. G. Didi-Huberman, 2002, p. 363-390. B. Gockel, 2007, p. 117-134.

54. Cf. U. Raulff, 1991, p. 55-70.

55. L. Binswanger et A. Warburg, 1924-1929, p. 257-258 (lettre du 28 décembre 1925).

56. Ibid., p. 274 (lettre du 16 décembre 1926).

57. Ibid., p. 280 (lettre du 18 juin 1927).

58. Ibid., p. 263-266 (lettre du 6 octobre 1926).

59. Ibid., p. 286 (lettre du $1^{\mathrm{er}}$ août 1927) et 292 (lettre du 18 juillet 1928).

60. Cf. C. Bologna, 2004, p. 281-282. E. Tavani, 2004, p. 121-143.

61. L. Binswanger et A. Warburg, 1924-1929, p. 252 (lettre du 23 décembre 1925).

62. A. Warburg, 1927a, p. 175-183.

INDEX

Mots-clés : Anamnèse, désastre de la guerre, psychomachie, psychiatrie, Binswanger, Denkraum, astra, monstra

\section{AUTEUR}

\section{GEORGES DIDI-HUBERMAN}

Philosophe et historien de l'art, enseigne à l'École des Hautes Études en Sciences Sociales (Paris). Il a séjourné à Rome (Académie de France), à Florence (Villa I Tatti-Harvard University Center for Italian Renaissance Studies), à Londres (Institute of Advanced Study, Warburg Institute). Il a enseigné dans de nombreuses universités étrangères (Johns Hopkins, Northwestern, Berkeley, Courtauld Institute, Berlin, Bâle...). Il a reçu deux prix de l'Académie des Beaux-Arts (Paris), le prix Hans Reimer de la Aby-Warburg-Stiftung (Hambourg), le Premio Napoli (Naples), le prix Humboldt (Berlin)... Il a dirigé plusieurs expositions, dont L'Empreinte au Centre Georges Pompidou (Paris, 1997), Fables du lieu au Fresnoy-Studio national des Arts contemporains (Tourcoing, 2001), Atlas au Museo Nacional Centro de Arte Reina Sofía (Madrid, 2010) et récemment Histoires de fantômes pour grandes personnes au Fresnoy avec Arno Gisinger.Il a publié une quarantaine d'ouvrages sur l'histoire et la théorie des images, notamment, pour les plus récents : La Ressemblance par contact. Archéologie, anachronisme et modernité de l'empreinte (Minuit, 2008) ; Quand les images prennent position. L'œil de l'histoire, 1 (Minuit, 2009). Remontages du temps subi. L'œil de l'histoire, 2 (Minuit, 2010) ; Atlas ou le gai savoir inquiet. L'œil de l'histoire, 3 (Minuit, 2011) ; Écorces (Minuit, 2011) ; Peuples exposés, peuples figurants. L'œil de l'histoire, 4 (Minuit, 2012). 